\title{
Violência sexual por parceiro íntimo identificada em Unidade Básica do PSF
}

RESUMO I Este estudo teve como objetivo identificar histórico de violência sexual e de sua associação com dados sociodemográficos, de saúde reprodutiva e sexual, entre as usuárias de três unidades do PSF. Metodologia: Estudo exploratório, transversal, população de 711 mulheres, entre 15 e 59 anos. Foi aplicado um questionário semi estruturado e utilizada a fórmula de Cochran para cálculo e amostra e o programa Statistical Package for Social Sciences (SPSS) para avaliar a associação entre a violência e as variáveis de interesse. Resultados: Encontrou-se uma população jovem em idade reprodutiva que vivia em união estável, escolaridade média de 9 anos, com atividade remunerada e renda de até dois salários mínimos. 12,9\% referiram violência sexual, o parceiro íntimo foi o principal agressor, número de gestações e de filhos esteve relacionado a ocorrência de violência sexual. Conclui-se que a freqüência de violência sexual foi alta, sendo o parceiro íntimo e o ex-parceiro, os principais agressores, apontando para a necessidade da investigação da violência como estratégia da saúde da família.

Palavras-chaves: Violência Sexual; Estupro; Unidade Básica de Saúde; Violência Contra a Mulher.

\begin{abstract}
This study aimed to identify a history of sexual violence and its association with sociodemographic, reproductive and sexual health data, among users of three PSF units. Methodology: Exploratory, transversal study, population of 711 women, between 15 and 59 years old. A semi-structured questionnaire was applied and the Cochran formula for calculation and sample and the Statistical Package for Social Sciences (SPSS) program were used to assess the association between violence and variables of interest. Results: We found a young population of reproductive age who lived in a stable union, with an average education of 9 years, with paid activity and income of up to two minimum wages. $12.9 \%$ reported sexual violence, the intimate partner was the main aggressor, number of pregnancies and children was related to the occurrence of sexual violence. It was concluded that the frequency of sexual violence was high, with the intimate partner and the ex-partner being the main aggressors, pointing to the need to investigate violence as a family health strategy.
\end{abstract}

Keywords: Sexual Violence; Rape; Basic Health Unit; Violence Against Women.

RESUMEN | Este estudio tuvo como objetivo identificar una historia de violencia sexual y su asociación con datos sociodemográficos, de salud reproductiva y sexual, entre los usuarios de tres unidades de PSF. Metodología: estudio exploratorio, transversal, población de 711 mujeres, entre 15 y 59 años. Se aplicó un cuestionario semiestructurado y se utilizó la fórmula de Cochran para el cálculo y la muestra y el programa Paquete estadístico para ciencias sociales (SPSS) para evaluar la asociación entre violencia y variables de interés. Resultados: encontramos una población joven en edad reproductiva que vivía en una unión estable, con una educación promedio de 9 años, con actividad remunerada e ingresos de hasta dos salarios mínimos. El 12.9\% reportó violencia sexual, la pareja íntima fue el agresor principal, el número de embarazos y niños estuvo relacionado con la ocurrencia de violencia sexual. Se concluyó que la frecuencia de la violencia sexual era alta, siendo la pareja íntima y la ex pareja los principales agresores, señalando la necesidad de investigar la violencia como una estrategia de salud familiar.

Descriptores: Violencia Sexual; Violación Unidad Básica de Salud; Violencia Contra la Mujer.

\section{Ana Cristina Fernandes Silva}

Enfermeira. Mestre em Saúde Pública. Hospital Perola Byington.

\section{Andrea Mathias Losacco}

Enfermeira. Mestrado em Epidemiologia. Enfermeira da Epidemiologia do Instituto de Infectologia Emílio Ribas. Docente da Graduação e Pós-Graduação de Enfermagem na UNISA.

\section{luri Abrahão Monteiro}

Engenheiro Elétrico. Universidade Federal de Ouro Preto.

\section{Anelise Riedel Abrahão}

Enfermeira, mestre em morfologia, doutora em enfermagem. Professora Associada da UNIFESP.

Recebido em: 09//03/2020

Aprovado em: 11/03/2020

INTRODUÇÃO

1 iolência sexual, conforme código penal, é constranger alguém, mediante violência ou grave ameaça, a ter conjunção carnal ou a praticar ou permitir que com ele se pratique outro ato libidinoso ${ }^{(1)}$.

No Brasil, estudo conduzido, em 2010, estimou que duas em cada cinco mulheres (40\%) já teriam sofrido algum tipo de violência ao menos uma vez na vida(2).

A questão da violência tem crescentemente chamado a atenção dos órgãos internacionais que, cada vez mais, identificam os efeitos das relações e situações de violência como um grande impeditivo para o desenvolvimento humano, refletindo na economia do país ${ }^{(3)}$.
A fundamentação da violência como problema de saúde pública não se faz somente pela magnitude das estatísticas envolvidas, mas considera os agravos para a saúde das pessoas por ela atingidas ${ }^{(4)}$.

Em artigo publicado no Informe Mundial sobre Violência e Saúde da Organização Mundial da Saúde (OMS), Dahlberg e Krug(5) afirmam que, embora muitas agressões não apresentem danos físicos, psicológicos e/ou sociais imediatos, podem levar a conseqüências tardias e perdurar por anos após o abuso inicial.

Conforme estudo do Banco Mundial, o estupro e a violência doméstica acometem $5 \%$ de anos de vida perdidos pela mulher em idade reprodutiva em países em desenvolvimento ${ }^{(6)}$.

É difícil avaliar o real impacto da 
violência sobre os serviços de saúde ou seus efeitos na produtividade econômica em todo o mundo. A evidência científica mostra que as mulheres, vítimas de violência doméstica e sexual, têm mais problemas de saúde, aumentando a demanda dos serviços de saúde ${ }^{(5,7)}$.

A violência acarreta uma série de efeitos na vida das mulheres, expressos por meio de uma complexidade de sentimentos e sequelas, como: o trauma emocional, o medo, os problemas físicos, a insônia, dificuldade em retomar a vida sexual e o trabalho, piora da saúde geral, dor crônica, abuso de substâncias, distúrbios reprodutivos e gravidez de alto risco causando danos psicológicos, com efeitos intensos e devastadores, por vezes, irreparáveis ${ }^{(8-11)}$.

Romper com o ciclo da violência é extremamente difícil para grande parte das mulheres que vivem nessa situação; muitas mulheres que tomam a atitude de denunciar seus companheiros agressores, muitas vezes, acabam desistindo de manter a denúncia(12).

Outros fatores também são responsáveis pela manutenção da relação de violência, como: as promessas do companheiro de não agredir mais, a falta de perspectiva e de condições materiais para um recomeço, a vergonha e a falta de apoio da família e da sociedade. Tudo isso acaba facilitando uma reconciliação, gerando a "rotinização da violência"(12).

Assim, a mudança desse cenário implica em uma importante estratégia ao dar voz às mulheres em situação de violência, para que, com base em seu conhecimento, valores e vivências traduzam as suas reais necessidades quando buscam por apoio e suporte das instituições ${ }^{(13)}$.

As unidades básicas de saúde são as portas de entrada para a população onde os profissionais estão mais próximos das mulheres e possuem melhores condições de acolhimento, com espaço para ouvir e entender e ajudar a enfrentar a violência de gênero ${ }^{(14)}$.

Objetivou-se identificar as características da violência sexual entre as usuárias em unidades básicas de saúde do Programa Saúde da Família (PSF)

\section{METODOLOGIA}

Estudo transversal exploratório, realizado com mulheres usuárias de três unidades básicas de saúde (UBS) do PSF na região do Jabaquara, município de São Paulo. A população do estudo foram mulheres usuárias e cadastradas nas respectivas UBS.

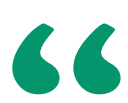

Romper com o
ciclo da violência é extremamente difícil para grande parte das mulheres que vivem nessa situação; muitas mulheres que tomam a atitude de denunciar seus companheiros agressores, muitas vezes, acabam desistindo de manter a denúncia ${ }^{(12)}$.

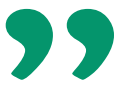

Os critérios de inclusão foram: mulheres com idade entre 15 e 59 anos, que concordaram em participar do estudo e assina- ram o Termo de Livre Consentimento (TLC).

O cálculo baseou-se no trabalho realizado por Silva ${ }^{(15)}$, em que se verificou uma frequência de violência contra a mulher de $27,5 \%$ na população do seu estudo. Para a determinação do número mínimo de participantes do estudo, foi realizado o cálculo de amostragem proposto por Cochran $^{(16)}$, considerando uma margem de erro de $10 \%$, foi estabelecida amostra mínima de 77 elementos amostrais.

Com o intuito de diminuir a margem de erro, além do número mínimo, adotou-se o critério tempo de coleta de dados. O número de indivíduos que compôs a população do estudo ficou assim distribuído: 362 mulheres no Jardim Lourdes, 254 no Vila Clara e 95 no Milton Santos.

A coleta de dados foi realizada de $2^{a}$ a sábado, intercalando as UBS durante 6 meses. Realizado pela própria autora, face a face, na sala de espera das unidades do estudo.

Foi utilizado instrumento com questões sobre o perfil pessoal, social: idade, religião, estado civil, raça, escolaridade, ocupação, renda familiar; sobre o perfil sexual, reprodutivo: idade do inicio da atividade sexual, número de parceiros, uso de método contraceptivo, doença sexual, gestações anteriores e abortos, sobre o inventario da violência (física, sexual e psicológica) e os autores da violência, tipo de intimidação, situação ou atividade na abordagem do agressor, perfil da assistência na unidade de saúde, assistência após a violência. O que influenciou a procura de assistência.

Para a análise dos dados, foi usado o programa Statistical Package for Social Sciences (SPSS), em sua versão 19.0, para a construção das tabelas e gráficos.

Para associar as variáveis categóricas nominais, foi aplicado o Teste da Razão de Verossimilhança e, para associar as variáveis categóricas de violência com as variáveis escalares, foi aplicado o Teste de Mann-Whitney.

Com o intuito de verificar o grau de associação entre as variáveis de interesse, foi adotado o nível de significância de 5\% $(0,050)$ para a aplicação dos testes estatísticos. 
O projeto foi aprovado pela Comissão de Ética da UNIFESP e da SMS. As entrevistas foram realizadas após a assinatura do Termo de Livre Consentimento pelas participantes.

\section{RESULTADOS}

Um terço da população tinha até 29 anos e vivia em união estável, metade era parda, tinha até 11 anos de estudo e atividade remunerada, 41,2\% com renda familiar de até dois salários, não se verificou relação significante entre estas características e a ocorrência de violência sexual.

Quanto aos antecedentes sexuais, a maioria das entrevistadas iniciou a atividade sexual entre 15 e 18 anos, 15,6\% teve a primeira relação até 14 anos, 28\% não utilizava método contraceptivo, 70,6\% referiu até 3 parceiros, $6 \%$ referiu doença sexual, sendo o HPV mais presente.

A frequência de violência sexual en- contrada neste estudo foi de $12,9 \%$ do total das entrevistadas. Não houve diferença estatística entre as três unidades.

$\mathrm{Na}$ análise univariada, a violência sexual teve relação significante entre mulheres que tiveram mais de 4 gestações e mais de 3 filhos, como observado no Quadro 1.

A doença sexual, aborto espontâneo e provocado e a idade de início da atividade sexual também tiveram relação significante com a ocorrência de violência sexual, como observado no Quadro 2.

O parceiro íntimo foi apontado em todas as unidades do estudo como o principal agressor.

No total de mulheres que informaram ter sofrido violência sexual, 6,6\% foi intimidada, sendo, a força física $(64,6 \%)$, o meio mais utilizado pelo agressor para consumar o ato, seguido de grave ameaça. Com relação ao local da violência, a residência foi citada por $55,3 \%$ das entrevistadas.

Quadro 1. Violência sexual associada às variáveis escalares: gestações e número de filhos. São Paulo, SP, Brasil, 2011.

\begin{tabular}{|l|c|c|c|c|}
\hline Variável & Violência sexual & N & Média & P \\
\hline \multirow{2}{*}{$\begin{array}{l}\text { No gestações (mais } \\
\text { de 4) }\end{array}$} & Não & 557 & 2,69 & \multirow{2}{*}{$<0,001$} \\
\cline { 2 - 4 } & Sim & 85 & 3,75 & \\
\cline { 2 - 4 } & Total & 642 & 2,83 & \multirow{2}{*}{$<0,001$} \\
\cline { 2 - 4 } $\begin{array}{l}\text { No filhos } \\
\text { (mais de 3) }\end{array}$ & Não & 523 & 2,25 & \\
\cline { 2 - 4 } & Sim & 80 & 3,03 & \\
\cline { 2 - 4 } & Total & 603 & 2,35 & \\
\hline
\end{tabular}

Quadro 2. Violência sexual associada a variáveis de antecedentes sexuais. São Paulo, SP, Brasil, 2011.

\begin{tabular}{|l|c|c|c|c|}
\hline \multirow{2}{*}{ VARIÁVEIS } & \multicolumn{4}{|c|}{ Violência sexual } \\
\hline \multirow{2}{*}{ Doença sexual } & Sim & não & total & P \\
\hline \multirow{2}{*}{ Aborto espontâneo } & 14 & 29 & 43 & 0,001 \\
\cline { 2 - 5 } & $32,60 \%$ & $67,40 \%$ & $100,00 \%$ & \\
\cline { 2 - 5 } & 27 & 140 & 167 & 0,001 \\
\hline \multirow{2}{*}{ Aborto provocado } & $16,20 \%$ & $83,80 \%$ & 100,00 & \\
\cline { 2 - 5 } & 12 & 10 & 22 & 0,001 \\
\hline Início de atividade sexual \\
\cline { 2 - 5 } (de 10 até 14 anos) & $54 \%$ & $46 \%$ & $100 \%$ & \\
\cline { 2 - 5 } & 23 & 82 & 105 & 0,013 \\
\hline
\end{tabular}

\section{DISCUSSÃO}

Apesar de estudos encontrarem associação positiva de fatores socioeconômicos com a violência sexual ${ }^{(10-20)}$, este estudo não evidenciou resultado semelhante. A provável razão desse achado deve-se ao fato de a amostra ser homogênea, apesar de ser composta por três unidades básicas em bairros diferentes, cada uma com suas especificidades.

Quanto à prevalência de violência sexual verificada nesse estudo, Schaiber et al(21). encontraram dado semelhante em estudo realizado em serviço de saúde primária, em que um terço das mulheres já viveu esse tipo de experiencia.

A violência sexual traz vários agravos à saúde da mulher, como o Banco Mundial ${ }^{(15)}$ estimou que o estupro e a violência doméstica acometem cerca de 5\% a 16\% dos anos de vida reprodutiva da mulher.

Com relação as variáveis sexual e reprodutiva, um estudo realizado em duas regiões no Brasil ${ }^{(22)}$, encontrou resultados semelhantes ao nosso onde o número de gestações e filhos expõem a mulher à violência. A dependência financeira, emocional, imaginária e social gerada pela existência de vários filhos, muitas vezes, justifica a permanência das mulheres na relação violenta, e a faz desistir de denunciar o parceiro ${ }^{(23)}$.

Além disso, existem outras barreiras que estão associadas a permanência da muIher nos relacionamentos violentos, como não considerar viver uma relação violen$\mathrm{ta}^{(10,19,24)}$, medo e vergonha de pedir ajuda ${ }^{(13)}$ e esperança que o agressor mude ${ }^{(25)}$.

Dados que vêm corroborar com o encontrado nesse estudo, onde parceiro íntimo é o principal agressor e a residência das entrevistadas foi o local da violência mais citado.

Este estudo encontrou relação significante da variável idade de início da atividade sexual de 10 a 14 com a ocorrência da violência sexual. A OMS afirma que a idade de 10 a 14 é considerada a pré-adolescência ${ }^{(26)}$, fase de grande transformação biopsicossocial, essas mudanças fazem com que a adolescente tenha a necessi- 
dade de se auto afirmar no mundo, de ter suas ideias respeitadas, ter mais autonomia, procuram mais emoções e ações ${ }^{(27)}$. Nesse sentido, sente a necessidade de fazer parte de grupos sociais com mesmos objetivos, se permitindo condutas que podem deixa-la vulnerável, como o uso de drogas ilícitas, a ingestão de bebidas alcoólicas e relacionamentos em ambiente

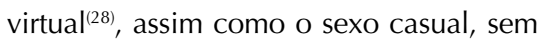
vínculo emocional, onde os direitos sexuais podem não ser respeitados.

É importante ressaltar, também, que nesta faixa etária a falta de maturidade e a vulnerabilidade física, psicológica e social são condições que facilitam a sedução e envolvimento com o agressor para a pratica e exploração sexual ${ }^{(29)}$. Mesmo que a relação sexual seja consentida, é considerado crime. O Código Penal Brasileiro, reformado recentemente, qualifica crime sexual contra vulnerável quando a vítima não é maior de 14 anos, por não ter discernimento para a prática do ato ou não poder de oferecer resistência ${ }^{(30)}$.

Esse estudo encontrou relação positiva da doença sexual com a ocorrência de violência sexual, a literatura mostra que o uso de preservativo masculino pode ser prejudicado nas relações violentas, em que as mulheres se sentem constrangidas em propor ao parceiro o seu uso, pois pode colocar em pauta a fidelidade da relação, e estar suscetível às Doenças Sexualmente Transmissíveis - DST ${ }^{(6)}$. O preservativo feminino ainda não faz parte da rotina da grande parte da população feminina devido algumas prerrogativas, a oferta na unidade de saúde básica e profissionais treinados, capazes de estimular e incentivar o uso do preservativo pela mulher ${ }^{(31)}$.

Resultados encontrados pela Pesquisa Nacional de Aborto (PNA) no inquérito realizado em 2010 revelaram que, a metade da população pesquisada que realizou aborto induzido, usou práticas não $\operatorname{seguras}^{(32)}$, fato que pode comprometer a saúde reprodutiva da mulher ${ }^{(33)}$, além de representar uma das principais causas de morte materna ${ }^{(34)}$.

Dentro desse entendimento, são vá- rios os riscos que as mulheres estão expostas, e a violência mostra-se como um facilitador na ocorrência desse evento, como risco duas vezes maior de aborto para mulheres em situação de violência(6).

As agressões podem causar lesões nas vítimas e provocar abortos espontâneos, além disso, a mulher, muitas vezes, opta em realizar aborto provocado por rejeitar o agressor e o concepto que dele foi gerado.

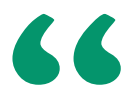

As agressões podem causar lesões nas vítimas e provocar abortos espontâneos, além disso, a mulher, muitas vezes, opta em realizar aborto provocado por rejeitar o agressor e o concepto que
dele foi gerado.

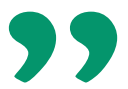

A violência sexual tem algumas implicações na vida da mulher, além dos traumas psicológicos, uso de drogas, comportamento de risco para doenças sexuais e perda do controle reprodutivo.

O estupro e a violência doméstica acometem cerca de $5 \%$ a $16 \%$ dos anos de vida reprodutiva da mulher(6). Mulheres agredidas sexualmente apresentam maior nível de gravidade de estresse pós-traumático; com forte associação aos sintomas depressivos e de ansiedade ${ }^{(35,36)}$ e pior classificação de saúde ${ }^{(37)}$.

Em relação à autoria das agressões, esse estudo encontrou o companheiro e ex-companheiro como os principais agressores, pesquisa realizada entre usuárias de serviço de saúde encontrou dado semelhante, com a prevalência de $44 \%$ de mulheres agredidas pelo parceiro durante a vida ${ }^{(38)}$.

Quando a violência ocorre em ambiente privado, faz com que a mulher, por questões culturais, sociais e religiosas, compreenda seu parceiro e respeite o vínculo afetivo e hierárquico de que compartilha ${ }^{(25)}$. Dessa forma, torna-se natural, habitual a convivência com certas situações violentas, presentes nos desdobramentos de seu cotidiano ${ }^{(33)}$, no qual os sentimentos de humilhação, vergonha e medo do parceiro facilitam a ocultação dos eventos violentos $^{(36)}$. Tendo como consequência a "invisibilização" da violência, a banalização e relativização dos atos violentos ${ }^{(10)}$, onde a mulher não se identifica como vítima nem reconhece tal ocorrido, como violação de seus direitos ${ }^{(24)}$.

\section{CONCLUSÃO}

Este estudo encontrou uma frequência de $12,9 \%$ de violência sexual em uma população de mulheres jovens, que vivem em união estável, com bom nível de escolaridade, sendo o parceiro íntimo o agressor mais frequente. Encontrou relação estatisticamente significante da variável violência sexual com as variáveis: início precoce de vida sexual (entre 10 e 14 anos de idade), mais de 4 gestações, mais de 3 filhos, doença sexual, aborto espontâneo.

O estudo evidenciou que a violência sexual está mais presente no ambiente familiar, reflexo da sociedade patriarcal, onde a mulher é a mais vulnerável nas relações de poder. A escolaridade não foi um fator protetor, demonstrando que a violência esta presente em todas as esferas da sociedade. A presença da violência, a submissão e a falta de conhecimento do 
seu próprio corpo limitam a autonomia da mulher, que não consegue exercer seus direitos reprodutivos, fazer suas escolhas, como mostra a relação positiva da violência sexual com as variáveis início de atividade sexual, número de gestação, número de filhos e doença sexual.

Este estudo permitiu a identificação de fatores relacionados à violência sexual por parceiro íntimo, importantes no enfrentamento da violência, para promover a sensibilização dos profissionais da saúde, para direcionar a implantação de novas estratégias e criar espaço para a mulher ser ouvida e acolhida.

\section{Referências}

1. Ministério da Saúde, Secretaria de Atenção à Saúde, Departamento de Ações Pragmáticas Estratégicas (BR). Aspectos jurídicos do atendimento às vítimas de violência sexual: perguntas e respostas para profissionais de saúde. 48p. 2. ed. Brasilia: Editora do Ministério da Saúde, 2011.

2.Venturi G, Recaman M, Oliveira S. A mulher brasileira no espaço público e privado. São Paulo: Fundação Perseu Abramo; 2010.

3. Banco Mundial. Relatório de Desenvolvimento Mundial de 1993: Investindo na Saúde. Nova lork, Oxford: University Press. Morrison A, Ellsberg M, Bott S, Como abordar la violência de gênero en la América Latina y el Caribe. Relatório do Banco Mundial Janeiro. 2005; 60.

4. Aiken M. False Alegation: a concept in the context of rape. J Psychosocial Nurs Ment Health Serv. 1993; 31:15-20.

5. Dahlberg LL, Krug, EG. Violência: um problema global de saúde pública. Ciênc. saúde coletiva. 2006; 11:1163-1178.

6. Heise L, Pitanguy J G. Violence Against Women: The Hidden Health Burden. World Bank Discussion Papers. 1994; 255.

7. Schraiber LB, Barros CRS, Castilho EA. Violência contra as mulheres por parceiros íntimos: usos de serviços de saúde. Rev. bras. epidemiol. 2010 Jun;13(2): 237-245.

8. Beebe DK. Sexual assault: the physician's role in prevention and treatment. J Miss State Assoc. 1998; 39:366-9.

9. Plichta SB. Intimate Partner Violence and Physical Health Consequences. J Interperson violence 2004; 19(11): 1296-323.

10. Kronbauer JFD, Meneghel SN. Perfil da violência de gênero perpetrada por companheiro. Rev. Saúde Pública. 2005 out; 39(5):695-701. 11. Oliveira $E M$, et al. Atendimento às mulheres vítimas de violência sexual: um estudo qualitativo. Rev. Saúde Pública. 2005 Jun; 39(3):376382.

12. Santi LN, Nakano AMS, Lettire A. Percepção de mulheres em situação de violência sobre o suporte e apoio recebido em seu contexto social Texto contexto - enferm. 2010 jul./set.; 19(3).

13. Miranda MPM, Paula CS, Bordin IA. Violência conjugal física contra a mulher na vida: prevalência e impacto imediato na saúde, trabalho e família. Rev Panam Salud Publica. 2010 abr; 27(4):300-308.

14. Moura LBA, et al. Violencias contra a mulheres por parceiro íntimo em área urbana economicamente vulnerável, Brasília, DF. Rev. Saúde Pública. 2009 dez; 43(6):944-953.

15. Silva MA, Falbo Neto GH, Figueiroa JN, Cabral Filho JE. Violence against women: prevalence and associated factors in patients attending a public healthcare service in the Northeast of Brazil. Cad. Saúde Pública 2010 feb; 26(2).

16. Cochran W. Sampling Techniques. 3. ed., New York: John Wiley \& Sons; 1986.

17. Oshikata $\mathrm{CT}$, Bedone Aloíso J, Faúndes A. Atendimento de emergência a mulheres que sofrem violência sexual: característica das muIheres e resultados até seis meses pós-agressão. Cad. Saúde Pública. 2005 jan-fev; 21(1): 192-199.

18. Jewkes R, Levin J, Penn-Kekana L. Risk factors for domestic violence: findings from a South African cross-sectional study. Social Science \& Medicine. 2002; 55:1603-1617.

19. Schraiber LB, D'Oliveira AFPL, França Junior I. Violência Sexual e mulheres no Brasil urbano, 2005. Rev. Saúde Pública. 2008 Jun; 42(suppl.1):127-137.
20. Vieira EM, Perdona GSC, Santos MA. Fatores associados à violência física por parceiro íntimo em usuárias de serviços de saúde. Rev. Saúde Pública. 2011; 45(4).

21. Schraiber LB, et al. Violência contra mulheres entre usuárias de serviços de saúde da Grande São Paulo. Rev. Saúde Pública. 2007; 3:359-367.

22. D'Oliveira AFPL, et al. Atenção integral à saúde de mulheres em situação de violência de gênero: uma alternativa para a atenção primária em saúde. Ciênc. saúde coletiva. 2009 ago; 14(4):1037-1050.

23. Jong LC, Sadala MLA, Tanaka ACD'A. Desistindo da denuncia ao agressor: relatao de mulheres vítimas de violência doméstica. Rev Esc Enferm USP. 2008; 42(4):744-751.

24. World Health Organization (WHO). Multi study on women's health and domestic violence against woman. Geneva: WHO; 2005.

25. Oshikata CT, et al. Características das mulheres violentadas sexualmente e da adesão ao segmento ambulatorial: tendências observadas ao longo dos anos em um serviço de referência em Campinas, São Paulo, Brasil. Cad. Saúde Pública. 2011; 27(4): 701-713.

26. World Health Organization (WHO). A Challenge for Society. Report of a WHO Study Group on Yong People and Health for All. Technical Report Series 731. Geneva: WHO, 1986.

27. Ministério da Saúde (BR). Lobato AL (org). Organização Pan Americana da Saude. Saúde e sexualidade de adolescentes. Construindo equidade no SUS. 2017. P.71.

28. Souza VP, et al. Fatores de risco associados à violência sexual. AV Enferm. 2019: 37(3): 364-374.

29. Souza CS, Costa MCO, Assis SG, Musse JO, Sobrinho CN, Amaral MTR. Sistema de vigilância e acidentes/ VIVA e a notificação de violência infanto-juvenil, no Sistema Único de Saúde - SUS de Feira de Santana - Bahia, Brasil. Ciênc Saúde Coletiva. 2014; 19: 773-84.

30. Barroso B, Araújo Junior MA (org). Vade Mecum OAB \& Concurso. 13. Edição. 2020. P. 2192.

31. Albuquerque GA, Alencar G. Autonomia sexual feminina: o preservativo feminino nas práticas eróticas. Saúde.com. 2015 ago; 11(2): 123-136.

32. Diniz D, Medeiros M. Abortion in Brazil: a household survey using the ballot box technique. Ciênc. saúde coletiva. 2010 Jun; 15(supp 1). 33. Hardy E, Alves G. Complicações Pós-Aborto Provocado: Fatores Associados. Cad Saúde Pública. 1992 out./dez.; 8 (4): 454-458.

34. Organización Panamericana de la Salud. Unidad de Género y Salud. Equidad de género y salud en las Américas a comienzos del siglo XXI. Washington: OPS; 2004.

35. Ilanes E, Bustos L, Vizcarra MB, Muñoz S. Violência y fatores sociales asociados a salud mental en mujeres de la ciudad de Temuco. Rev Méd Chile. 2007; 135: 326-334

36. Sarasua B, Zubizarreta I, Echeburúa ECP. Perfil psicopatológico diferencial de las víctimas de violencia de pareja en función de la edad. Psicothema 2007; 19(3):459-466.

37. Bonomi A, et al. Health Outcomes in Women with Physical and Sexual Intimate Partner Violence Exposure Journal of Women's Health. 2007; 16(7).

38. Thompson RS, et al Intimate Partner Violence: Prevalence, Types, and Chronicity in Adult Women. American Journal of Preventive Medicine. 2006 Jun; 30(6):447-457. 